\title{
Role of solvation structure in the shuttling of the hydrated excess proton $^{\dagger}$
}

\author{
RAJIB BISWAS and GREGORY A VOTH* \\ Department of Chemistry, James Franck Institute, and Institute for Biophysical Dynamics \\ The University of Chicago, Chicago, IL 60637, USA \\ E-mail: gavoth@uchicago.edu
}

MS received 9 February 2017; revised 14 April 2017; accepted 18 April 2017

\begin{abstract}
The classic Marcus electron transfer reaction model demonstrated that a barrierless electron transfer reaction can occur when both the reactant and product have almost similar solvation environment. In our recently developed proton model, we have incorporated the pre-solvation concept and showed that it indeed facilitates the proton diffusion in aqueous solution. In this work, we further quantify the degree of pre-solvation using different structural parameters, e.g., tetrahedral order parameter, average numbers of hydrogen bonds. All the above said parameters exhibit a very strong correlation with the proton share parameter. The more Zundel-like configurations have almost identical solvation environment for both the water molecules and support the presolvation concept. However, in the case of Eigen-like configurations, the central hydronium and "special pair" water have distinctly different solvation structures.
\end{abstract}

Keywords. Empirical valence bond; reactive molecular dynamics; pre-solvation; proton transfer; proton transport.

\section{Introduction}

The aqueous transfer or "shuttle" of a hydrated excess proton between water molecules is extremely important to explore many important processes involved in biological $^{1-4}$ and energy systems. ${ }^{5-8}$ In aqueous solution, an excess proton can interact with the surrounding water in a very fast time scale. The interaction strength can span continuously between a strong covalent interaction and a weaker hydrogen bond, which leads to the delocalization of excess positive charge defect. These excess proton water solvation structures can be by and large defined by a continuous evolution of different transient configurations within two limiting forms, the Eigen cation ${ }^{9}$ (hydronium solvated with three water molecules) and Zundel cation ${ }^{10}$ (proton equally shared between two water molecules). The fast transport of excess charge in case of the aqueous hydrated excess proton occurs via the Grotthuss mechanism, ${ }^{3,11,12}$ in which the excess proton is transferred by the breaking and forming of chemical bonds. Because of the rapid time scale and strong coupling of the structural evolution with proton

\footnotetext{
*For correspondence

${ }^{\dagger}$ Dedicated to the memory of the late Professor Charusita Chakravarty.
}

transfer process, it is extremely challenging to study the process experimentally and computationally as well.

Despite several challenges, the excess proton solvation and transport have been studied extensively by experiments ${ }^{13-19}$ and simulations. ${ }^{4,20-44}$ Ultrafast high-resolution spectroscopy ${ }^{13-19}$ and advanced computer simulation techniques, ${ }^{4,6-8,20-44}$ have enhanced our understanding of the proton transport mechanisms, particularly in the case of aqueous systems. The dynamical evolution of bonding topologies in terms of reorganization of water hydrogen bond (H-bond) network requires special attention for building a reasonable model to study proton transfer in aqueous solution. Because of the absence of bond breaking and bond formation features, classical molecular dynamics simulation does not allow to study proton solvation and transfer. On the other hand, in the case of $a b$ initio molecular dynamics (AIMD) the explicit treatment of electronic degrees of freedom by the system Hamiltonian makes it one possible choice to study proton solvation and charge transfer more accurately. ${ }^{20,22,24,25,27,29,33,35,37,41-43,45}$ However, the AIMD method comes with a very high computational cost which, thus, limits the system size and time scales that can be studied. In addition to this, the intrinsic approximations accompanying with the level of density functional theory (DFT) employed in AIMD can also 
give rise to unphysical consequences, particularly in the case of liquid water. ${ }^{27,43,46,47}$

However, despite these facts, the inherent capability of treating electronic polarization, chemical bondbreaking, and charge transfer by AIMD can provide significant information for parameterization of more efficient computational models. ${ }^{36,39}$ One such alternate approach based on the Multistate Empirical Valence Bond (MS-EVB) formalism has been shown to simulate excess proton solvation and transfer successfully while accurately capturing the essential physics and chemistry in different protonated systems..$^{4,23,26,31,40}$ The MS-EVB and AIMD studies together find that the Eigen cation is the predominant solvation structure in bulk water and that proton transfer happens via an Eigen-Zundel-Eigen mechanism. In case of Eigen configurations, it has been found from the earlier simulation studies that one of the three water ligands in its first solvation shell always prefers to be closer to the hydronium moiety than the other two and is termed as "special pair" water. ${ }^{20,32}$ It has also been found that before the proton transfer event, the "special pair" water interchanges its identity rapidly within the three first shell water molecules (the "special pair" dance). ${ }^{32,34}$

Recent AIMD data and the latest version of the MS-EVB model (version 3.2) both showed that "presolvation" (i.e., the hydronium is transiently solvated by four water molecules by accepting a weak hydrogen bond from the fourth water molecule) facilitates the proton shuttling and diffusion in aqueous solution. ${ }^{33,37,43,48}$ Since a water molecule typically forms four hydrogen bonds, in the pre-solvation picture, before the proton transfer happens, hydronium will also form four hydrogen bonds. This will create a similar solvation environment for both the hydronium and its nearby water molecules. ${ }^{33,37,43}$ Thus, if the excess proton transfers from hydronium to any one of its nearest water molecules in the pre-solvated condition, the newly formed water molecule will have similar coordination number as in the bulk water. This pre-solvation concept can be well understood by the classic Marcus picture of proton transfer. ${ }^{49,50}$ In the Marcus picture, the free energy of the system along the (slow) solvent coordinate is similar before and after a proton transfer reaction so that the transition can happen in the (fast) proton transfer coordinate. In the present work, we further quantify the degree of pre-solvation using different structural parameters, e.g., a tetrahedral order parameter and average hydrogen bonds of proton donor and acceptor moieties.

The remaining sections of this paper are as follows: In section 2, we briefly discuss the method and the simulation details. Section 3 contains the main results of the present work, and conclusions are given in section 4 .

\section{Computational/theoretical}

\subsection{Theoretical framework of the MS-EVB model}

It is a well-established fact that the delocalized nature of the excess proton charge or charge defect can be described by the MS-EVB approach in an efficient and accurate way. ${ }^{23,26,31,38}$ The complete description of the MS-EVB methodology can be found in earlier papers. ${ }^{23,26,31}$ In this article, we summarize the few key features and advances in the case of the latest version i.e., MS-EVB 3.2.

In the MS-EVB formalism, for a given nuclear configuration, the ground state of the system $|\psi\rangle$ is represented by a linear combination of different "basis states" $|i\rangle$ corresponding to different bonding topologies:

$$
|\psi\rangle=\sum_{i=1}^{N} c_{i}|i\rangle
$$

where $N$ is the total number of chemically motivated basis states and $c_{i}$ 's are the EVB coefficientsof $i^{\text {th }}$ basis states. The coefficients $\left(c_{i}\right)$ can be obtained by solving the eigenvalueeigenfunction problem using the Hamiltonian matrix $\mathbf{H}$, which comprises of matrix elements $h_{i j}=\langle i|\mathbf{H}| j\rangle$, such that,

$$
\mathbf{H c}=E_{0} \mathbf{c}
$$

Here, $E_{0}$ is eigenvalue corresponding to the ground state energy; $\mathbf{c}$ represents eigenvector whose components are the EVB coefficients as shown in Eq. (1). The diagonal elements $h_{i i}$, contain terms that are, e.g., (though not necessarily ${ }^{39}$ ) described by classical force fields ${ }^{51}$ and are expressed as,

$$
\begin{aligned}
h_{i i}= & V_{\mathrm{H}_{3} \mathrm{O}^{+}}^{\text {intra }}+\sum_{k=1}^{N_{\mathrm{H}_{2} \mathrm{O}}} V_{\mathrm{H}_{2} \mathrm{O}}^{\text {intra }, k}+\sum_{k=1}^{N_{\mathrm{H}_{2} \mathrm{O}}} V_{\mathrm{H}_{3} \mathrm{O}^{+}, \mathrm{H}_{2} \mathrm{O}}^{\text {inter }, k} \\
& +\sum_{k<k^{\prime}}^{N_{\mathrm{H}_{2} \mathrm{O}}} V_{\mathrm{H}_{2} \mathrm{O}}^{\text {inter }, k k^{\prime}}
\end{aligned}
$$

In addition to the functional forms of the intramolecular potential for hydronium $\left(V_{\mathrm{H}_{3} \mathrm{O}^{+}}^{\text {intra }}\right)$ and the intra- and intermolecular potentials for water $\left(V_{\mathrm{H}_{2} \mathrm{O}}^{\mathrm{intra} k}, V_{\mathrm{H}_{2} \mathrm{O}}^{\mathrm{inter}} k k^{\prime}\right)$ similar to those of MS-EVB 3.0 and SPC/Fw, ${ }^{31,52}$ in the latest excess proton model, MS-EVB 3.2, ${ }^{48}$ the intermolecular potential between water and hydronium, $V_{\mathrm{H}_{3} \mathrm{O}^{+}, \mathrm{H}_{2} \mathrm{O}}^{\mathrm{inte},}$ has an additional Lennard-Jones (LJ) interaction between the hydronium oxygen and water hydrogens to incorporate the pre-solvation concept, i.e., to account for the weak hydrogen bond acceptor nature of hydronium oxygen as suggested by recent AIMD results. ${ }^{33,43}$

To explore the effects of anharmonicity in the water $\mathrm{O}-\mathrm{H}$ stretching modes on the structural and dynamical properties, particularly on the vibrational spectrum of the hydrated excess proton, we have also developed the anharmonic version of the latest proton model i.e., aMS-EVB 3.2. ${ }^{48}$ It is based on the flexible anharmonic water model aSPC/Fw developed by Park et $a{ }^{40}$ In addition to the MS-EVB 3.2 model, in this work, 
we have quantified the degree of pre-solvation in aMS-EVB 3.2 model as well.

\subsection{Simulation details}

The reactive molecular dynamics simulations using the MSEVB 3.2 excess proton model were performed using a modified version of the LAMMPS ${ }^{53}$ MD code. The simulation box consisted of $256 \mathrm{SPC} / \mathrm{FW}^{52}$ water molecules with an excess proton and a chloride anion. The parameters for chloride ion were obtained from Dang. ${ }^{54}$ The system is first equilibrated in a constant $N V T$ ensemble by keeping the density and temperature at $1.0 \mathrm{~g} / \mathrm{cm}^{3}$ and $298 \mathrm{~K}$. The temperature was kept constant by using a Nosé-Hoover chain of three thermostats, ${ }^{55}$ along with 0.5 fs time step and a relaxation time of 0.05 ps. The long-range electrostatics were calculated using Ewald summation with a relative error of $10^{-5}$ in forces. ${ }^{56}$ The cutoff value for the LJ interactions was set to 9 $\AA$. Finally, the production data were collected with a constant NVE dynamics for a total length of $10 \mathrm{~ns}$ as obtained from 10 independent 1-ns long trajectories. In the case of aMS-EVB 3.2 model, we follow the same simulation strategy except in this case we use aSPC/Fw ${ }^{40}$ water model.

\section{Results and Discussion}

The pre-solvation concept suggests that when two water molecules forming the special pair by solvating the excess proton structure have identical solvation environment, the proton can smoothly transfer from one water molecule to the other. This pre-solvation idea is very much similar to the Marcus picture of proton transfer, ${ }^{49,50}$ in which the free energy of the system along the solvent coordinate is comparable before and after a proton transfer event so that the transition can happen very easily in the proton transfer coordinate. In our earlier studies, we have shown that though the gas phase energy scan reflects an indication of the pre-solvation scenario, ${ }^{57}$ it is not able to explain the exact path for the bulk phase. ${ }^{43}$ The reactive molecular dynamics using MS-EVB approach can, however, capture the proton transfer events and the dynamical evolution of the solvation structures as well. In the case of reactive molecular dynamics simulation, after a proton transfer event, the acceptor water molecule can become hydronium moiety by accepting the excess proton. Thus, the atom index of each atom in the newly formed hydronium will be different from that of the old hydronium. In Figure 1, we show the evolution of hydronium-like oxygen atom index in case of the MS-EVB 3.2 proton model. It is evident from the figure that the excess proton is fluctuating from one water molecule to another in very fast time scale.
In order to gain insight about the pre-solvation concept, we first examine the proton sharing parameter, $\delta$, as shown in Figure 2, and define a $\delta$-dependent radial distribution function of hydronium oxygen and water hydrogen (shown in Figure 3). It is clear from the figure, that in the case of small $\delta$, the radial distribution function shows small intensity around $r \sim 2.0 \AA$, which corresponds to the fourth water molecules approaching towards hydronium oxygen.

To further quantify the solvation environment of the excess positive charge donor (hydronium) and the excess charge acceptor (special pair water), we analyze the local tetrahedral order parameter of the hydronium moiety and the special pair water separately. The local tetrahedral order parameter is described as, ${ }^{58}$

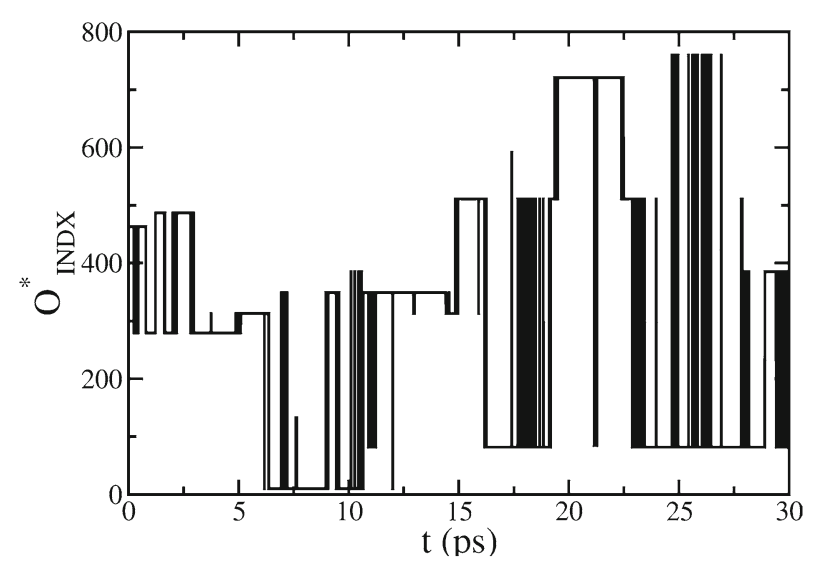

Figure 1. Time evolution of the oxygen index of the most hydronium-like oxygen atom, i.e., the most dominant EVB states in case of MS-EVB3.2.

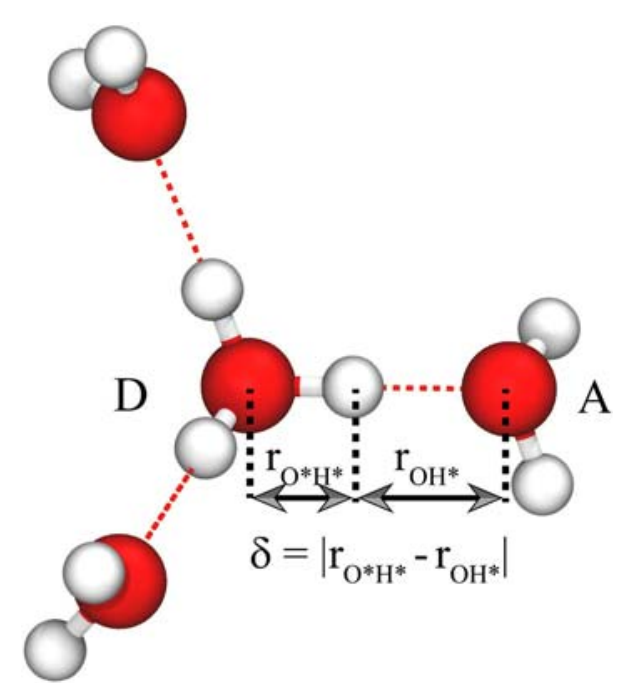

Figure 2. Excess charge donor (D), i.e., the hydronium moiety, excess charge acceptor (A), i.e., special pair water and the definition of proton sharing parameter $\delta$. 


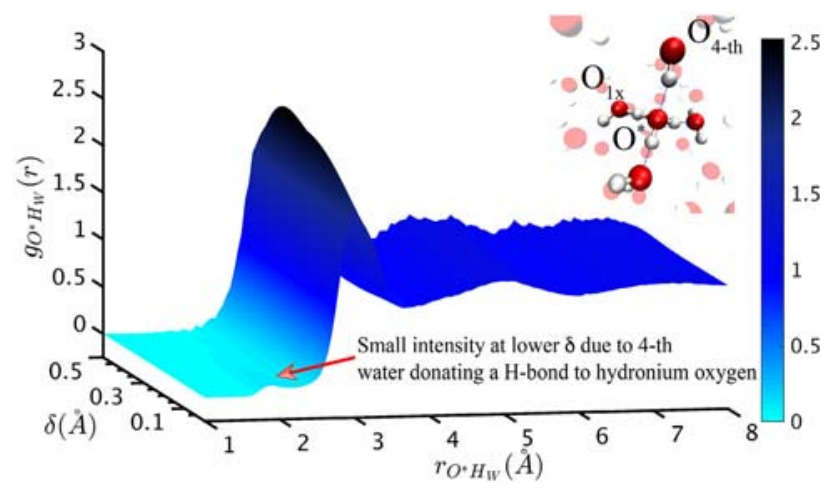

Figure 3. The proton sharing parameter ( $\delta$ )-dependent radial distribution function of the most hydronium-like oxygen $\left(\mathrm{O}^{*}\right)$ and water hydrogen $\left(\mathrm{H}_{W}\right)$. The pre-solvation is reflected in terms of small intensity near $r \sim 2.0 \AA$ for low $\delta$ which represents the $4^{\text {th }}$ water molecule donating a $\mathrm{H}$-bond to the hydronium oxygen.

$$
t_{h} \equiv 1-\frac{3}{8} \sum_{j=1}^{3} \sum_{k=j+1}^{4}\left(\cos \psi_{j k}+\frac{1}{3}\right)^{2} .
$$

where, $\psi_{j k}$ is the angle formed by the lines joining the oxygen atom of the molecule under consideration and its nearest two oxygen atoms $j$ and $k$. For perfect tetrahedral arrangement, $t_{h}$ reaches its maximum value of unity and for a non-interacting system, and the average value of $t_{h}$ is zero.

In Figure 4, we show the angle distributions for both the excess proton models in case of $\delta<0.1 \AA$ and $\delta>0.4 \AA$. It is clear from the figure that in the case of Zundel-like configurations (i.e., $\delta<0.1 \AA$ ) both the hydronium ion and the special pair water have almost identical solvation environment. However, in the case of the Eigen-like configurations the angle distributions of hydronium moiety are distinctly different from that of the special pair water. We also find that the average $t_{\mathrm{h}}$ values of hydronium, and special pair water molecules for Zundel-like configurations are almost comparable (for MS-EVB 3.2 it is 0.72 vs. 0.69 and in the case of aMS-EVB 3.2, it is 0.71 vs. 0.68 ).

To further quantify the local structure around the donor oxygen and acceptor oxygen (as defined in Figure 2), we calculate the distribution of total hydrogen bond (H-bond) number for each center. We use a geometrical definition of $\mathrm{H}$-bond, i.e., the tagged proton of the $\mathrm{O}-\mathrm{H}$ bond of interest is considered to have a hydrogen bond if intermolecular oxygen distance is less than $3.5 \AA$, and the angle between the intermolecular $\mathrm{O}-\mathrm{O}$ distance vector and the $\mathrm{O}-\mathrm{H}$ bond vector is less than $30^{\circ} .{ }^{59}$ In Figure 5, we represent the hydrogen bond
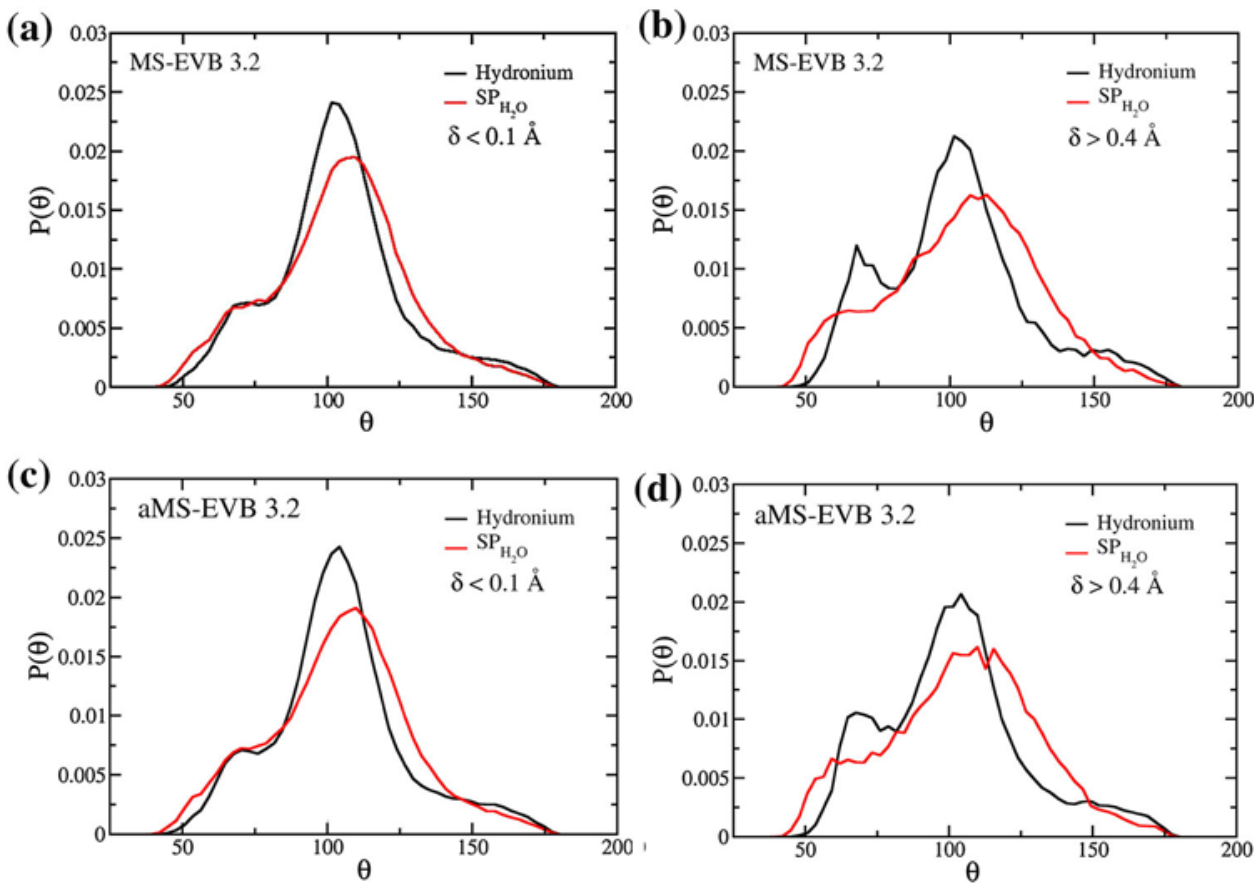

Figure 4. Angle distributions for tetrahedral order parameter in case of excess proton donor (hydronium) is shown in black and in case of excess proton acceptor (the special pair water) is represented in red. Top panel shows the results for the MS-EVB 3.2 and bottom panel for the aMS-EVB 3.2 proton models. Results for the configurations having $\delta<0.1 \AA$ are shown in (a) and (c); and, the configurations having $\delta>0.4 \AA$ are represented in (b) and (d). 

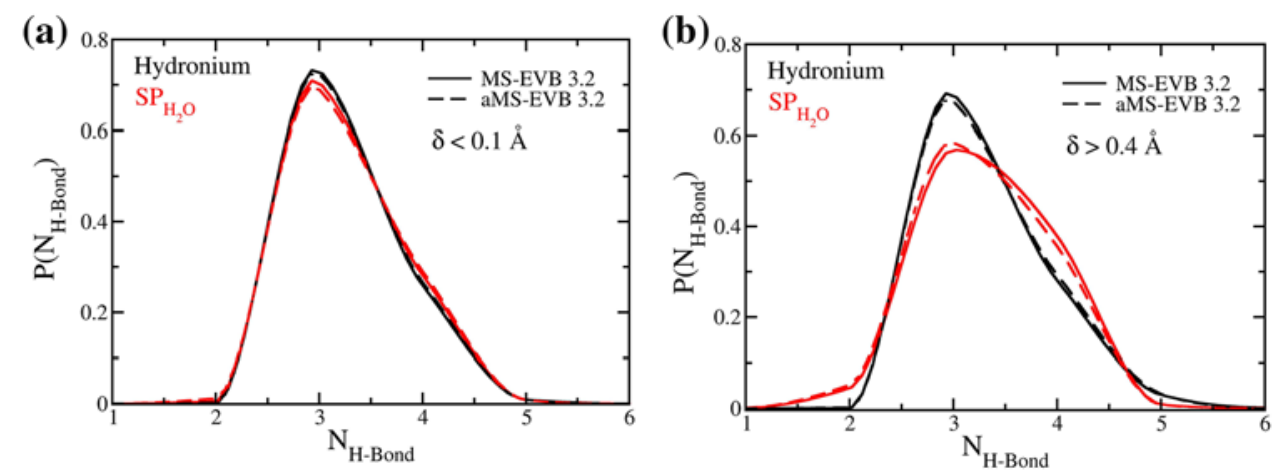

Figure 5. Distribution of number of H-bond in case of, (a) Zundel-like configurations (structure with $\delta<0.1 \AA$ ), and (b) Eigen-like configurations with $\delta>0.4 \AA$. For both the proton models in case of Zundel-like configurations hydronium and special pair water have similar number of H-bonds. However, in case of Eigen-like configurations the scenario is completely different.

number distribution in the case of Zundel-like configurations and Eigen-like configurations having $\delta>0.4 \AA$. It is evident from Figure 5(a) that in the Zundel-like configurations hydronium and special pair water have identical H-bond distributions. This reflects that when two water molecules equally share the proton, both the water molecules are experiencing similar solvation environments and as a result proton can transfer from one water molecule to the other by experiencing very small barrier. Thus, a pre-defined solvent orientation is highly coupled with the proton transfer events. This observation becomes more evident when we study the H-bond distributions for configurations with $\delta>0.4 \AA$ (shown in Figure 5(b)). From Figure 5(b) it is clear that when the excess charge is more localized on the hydronium, the special pair water and hydronium have completely different local structure.

\section{Conclusions}

In our earlier work, we have already shown that the presence of $4^{\text {th }}$ water molecule, weakly $\mathrm{H}$-bonded to the hydronium-like oxygen, actually facilitates the aqueous phase proton transfer process. In this work, we further quantify the extent of pre-solvation in proton transfer events by studying the solvent orientation and hydrogen bond numbers. The latest two new MS-EVB excess proton models have been studied for the presolvation picture. First, with the help of a proton sharing parameter-dependent ( $\delta$-dependent) radial distribution function, we show that the hydronium can indeed behave as a weak hydrogen bond acceptor. Furthermore, the local tetrahedral order parameter and angle distribution both suggest that, in Zundel configurations, the hydronium and special pair water have almost identical solvation structure. A similar conclusion is also supported by the $\delta$-dependent $\mathrm{H}$-bond distributions.

We therefore conclude from the present work that the hydrated excess proton transfer events in liquid water are even more highly coupled with local solvent orientations than previously thought. These results, while interesting in their own right, will also allow us to develop even more accurate reactive MD models and methods in the future for the excess proton in aqueous media.

\section{Acknowledgements}

This research was supported in part by the Department of Energy (DOE), Office of Basic Energy Sciences (BES), Division of Chemical Sciences, Geosciences, and Biosciences, (DOE-BES Grant No. DE-SC0005418), and by the National Science Foundation (NSF Grant No. CHE-1465248). The computational resources in this work were provided by the University of Chicago Research Computing Center (RCC).

\section{References}

1. Decoursey T E 2003 Voltage-gated proton channels and other proton transfer pathways Physiol. Rev. 83475

2. Wraight C A 2006 Chance and design - Proton transfer in water, channels and bioenergetic proteins $B B A$ Bioenergetics 1757886

3. Cukierman S 2006 Et tu, Grotthuss! and other unfinished stories BBA-Bioenergetics 1757876

4. Swanson J M J, Maupin C M, Chen H, Petersen M K, Xu J, Wu Y and Voth G A 2007 Proton Solvation and Transport in Aqueous and Biomolecular Systems: Insights from Computer Simulations J. Phys. Chem. B 1114300

5. Kreuer K D, Paddison S J, Spohr E and Schuster M 2004 Transport in proton conductors for fuel-cell applications: Simulations, elementary reactions, and phenomenology Chem. Rev. 1044637 
6. Jorn R, Savage J and Voth G A 2012 Proton Conduction in Exchange Membranes across Multiple Length Scales Acc. Chem. Res. 452002

7. Tse Y-L S, Herring A M, Kim K and Voth G A 2013 Molecular Dynamics Simulations of Proton Transport in $3 \mathrm{M}$ and Nafion Perfluorosulfonic Acid Membranes $J$. Phys. Chem. C 1178079

8. Savage J, Tse Y-L S and Voth G A 2014 Proton Transport Mechanism of Perfluorosulfonic Acid Membranes J. Phys. Chem. C 11817436

9. Eigen M 1964 Proton Transfer, Acid-Base Catalysis, and Enzymatic Hydrolysis. Part I: ELEMENTARY PROCESS Angew. Chem. Int. Edit. 31

10. Zundel G 2000 Hydrogen Bonds with Large Proton Polarizability and Proton Transfer Processes in Electrochemistry and Biology Adv. Chem. Phys. 1111

11. von Grotthuss C J T 1806 Sur la décomposition de l'eau et des corps qu'elle tient en dissolution à l'aide de l'électricité galvanique Ann. Chim. 5854

12. Agmon N 1995 The Grotthuss Mechanism Chem. Phys. Lett. 244456

13. Garczarek F and Gerwert K 2006 Functional waters in intraprotein proton transfer monitored by FTIR difference spectroscopy Nature 439109

14. Roberts S T, Petersen P B, Ramasesha K, Tokmakoff A, Ufimtsev I S and Martinez T J 2009 Observation of a Zundel-like transition state during proton transfer in aqueous hydroxide solutions Proc. Natl. Acad. Sci. U.S.A. 10615154

15. Roberts S T, Ramasesha K, Petersen P B, Mandal A and Tokmakoff A 2011 Proton Transfer in Concentrated Aqueous Hydroxide Visualized Using Ultrafast Infrared Spectroscopy J. Phys. Chem. A 1153957

16. Fayer M D 2013 Ultrafast Infrared Vibrational Spectroscopy (Boca Raton, Florida: CRC Press)

17. Mandal A, Ramasesha K, De Marco L and Tokmakoff A 2014 Collective vibrations of water-solvated hydroxide ions investigated with broadband 2DIR spectroscopy $J$. Chem. Phys. 140204508

18. Fournier J A, Johnson C J, Wolke C T, Weddle G H, Wolk A B and Johnson M A 2014 Vibrational spectral signature of the proton defect in the three-dimensional $\mathrm{H}+(\mathrm{H} 2 \mathrm{O}) 21$ cluster Science 3441009

19. Thämer M, De Marco L, Ramasesha K, Mandal A and Tokmakoff A 2015 Ultrafast 2D IR spectroscopy of the excess proton in liquid water Science $\mathbf{3 5 0} 78$

20. Tuckerman M, Laasonen K, Sprik M and Parrinello M 1995 Ab-Initio Molecular-Dynamics Simulation of the Solvation and Transport of Hydronium and Hydroxyl Ions in Water J. Chem. Phys. 103150

21. Lobaugh J and Voth G A 1996 The Quantum Dynamics of an Excess Proton in Water J. Chem. Phys. 104 2056

22. Tuckerman M E, Marx D, Klein M L and Parrinello M 1997 On the Quantum Nature of the Shared Proton in Hydrogen Bonds Science 275817

23. Schmitt U W and Voth G A 1999 The Computer Simulation of Proton Transport in Water J. Chem. Phys. 111 9361

24. Marx D, Tuckerman M E, Hutter J and Parrinello M 1999 The Nature of the Hydrated Excess Proton in Water Nature 397601
25. Tuckerman M E, Marx D and Parrinello M 2002 The nature and transport mechanism of hydrated hydroxide ions in aqueous solution. Nature $\mathbf{4 1 7} 925$

26. Day T J F, Soudackov A V, Cuma M, Schmitt U W and Voth G A 2002 A Second Generation Multistate Empirical Valence Bond Model for Proton Transport in Aqueous Systems J. Chem. Phys. 1175839

27. Izvekov $\mathrm{S}$ and Voth $\mathrm{G} A 2005$ Ab initio moleculardynamics simulation of aqueous proton solvation and transport revisited J. Chem. Phys. 123044505

28. Lapid H, Agmon N, Petersen M K and Voth G A 2005 A bond-order analysis of the mechanism for hydrated proton mobility in liquid water J. Chem. Phys. 122014506

29. Marx D 2006 Proton transfer 200 years after von Grotthuss: Insights from ab initio simulations. ChemPhysChem 71848

30. Chandra A, Tuckerman M E and Marx D 2007 Connecting solvation shell structure to proton transport kinetics in hydrogen-bonded networks via population correlation functions Phys. Rev. Lett. 99145901

31. Wu Y J, Chen H N, Wang F, Paesani F and Voth G A 2008 An improved multistate empirical valence bond model for aqueous proton solvation and transport J. Phys. Chem. B 112467

32. Markovitch O, Chen H, Izvekov S, Paesani F, Voth G A and Agmon N 2008 Special pair dance and partner selection: Elementary steps in proton transport in liquid water J. Phys. Chem. B 1129456

33. Berkelbach T C, Lee H S and Tuckerman M E 2009 Concerted Hydrogen-Bond Dynamics in the Transport Mechanism of the Hydrated Proton: A First-Principles Molecular Dynamics Study Phys. Rev. Lett. 103238302

34. Swanson J M J and Simons J 2009 Role of Charge Transfer in the Structure and Dynamics of the Hydrated Proton J. Phys. Chem. B 1135149

35. Marx D, Chandra A and Tuckerman M E 2010 Aqueous Basic Solutions: Hydroxide Solvation, Structural Diffusion, and Comparison to the Hydrated Proton Chem. Rev. 1102174

36. Knight C, Maupin C M, Izvekov S and Voth G A 2010 Defining Condensed Phase Reactive Force Fields from ab Initio Molecular Dynamics Simulations: The Case of the Hydrated Excess Proton J. Chem. Theory Comput. 6 3223

37. Tuckerman M E, Chandra A and Marx D 2010 A statistical mechanical theory of proton transport kinetics in hydrogen-bonded networks based on population correlation functions with applications to acids and bases $J$. Chem. Phys. 133124108

38. Knight C and Voth G A 2012 The Curious Case of the Hydrated Proton Acc. Chem. Res. 45101

39. Knight C, Lindberg G E and Voth G A 2012 Multiscale reactive molecular dynamics J. Chem. Phys. 13722 A525

40. Park K, Lin W and Paesani F 2012 A Refined MS-EVB Model for Proton Transport in Aqueous Environments $J$. Phys. Chem. B 116343

41. Hassanali A, Giberti F, Cuny J, Kuhne T D and Parrinello M 2013 Proton transfer through the water gossamer Proc. Natl. Acad. Sci. U.S.A. 11013723

42. Hassanali A A, Giberti F, Sosso G C and Parrinello M 2014 The role of the umbrella inversion mode in proton diffusion Chem. Phys. Lett. 599133 
43. Tse Y L, Knight C and Voth G A 2015 An analysis of hydrated proton diffusion in ab initio molecular dynamics J. Chem. Phys. 142014104

44. Peng Y, Swanson J M J, Kang S-g, Zhou R and Voth G A 2015 Hydrated Excess Protons Can Create Their Own Water Wires J. Phys. Chem. B 1199212

45. Marx D and Hutter J 2009 In Ab Initio Molecular Dynamics: Basic Theory and Advanced Methods (New York: Cambridge University Press)

46. Yoo S, Zeng X C and Xantheas S S 2009 On the phase diagram of water with density functional theory potentials: The melting temperature of ice I-h with the PerdewBurke-Ernzerhof and Becke-Lee-Yang-Parr functionals J. Chem. Phys. 130221102

47. Yoo S and Xantheas S S 2011 Communication: The effect of dispersion corrections on the melting temperature of liquid water J. Chem. Phys. 134121105

48. Biswas R, Tse Y L, Tokmakoff A and Voth G A 2016 Role of Presolvation and Anharmonicity in Aqueous Phase Hydrated Proton Solvation and Transport J. Phys. Chem. B 1201793

49. Ando K and Hynes J T 1997 Molecular Mechanism of $\mathrm{HCl}$ Acid Ionization in Water: Ab initio Potential Energy Surfaces and Monte Carlo Simulations J. Phys. Chem. B 10110464

50. Ando K and Hynes J T 1999 Acid-Base Proton Transfer and Ion Pair Formation in Solution Adv. Chem. Phys. 110 381
51. All the terms in a diagonal element are described by the classical force fields, with the exception of the intermolecular term between hydronium and water in which contains two extra "repulsive terms". See the original MS-EVB3 paper

52. Wu Y J, Tepper H L and Voth G A 2006 Flexible simple point-charge water model with improved liquid-state properties J. Chem. Phys. 124024503

53. Plimpton S 1995 Fast Parallel Algorithms for ShortRange Molecular Dynamics J. Comp. Phys. 1171

54. Dang L X 1995 Mechanism and Thermodynamics of Ion Selectivity in Aqueous-Solutions of 18-Crown-6 Ether - a Molecular-Dynamics Study J. Am. Chem. Soc. 117 6954

55. Martyna G J, Klein M L and Tuckerman M 1992 Nose-Hoover Chains - the Canonical Ensemble Via Continuous Dynamics J. Chem. Phys. 972635

56. Allen M P and Tildesley D J 1989 In Computer Simulation of Liquids (New York: Oxford University Press)

57. Jagoda-Cwiklik B, Cwiklik L and Jungwirth P 2011 Behavior of the Eigen Form of Hydronium at the Air/Water Interface J. Phys. Chem. A 1155881

58. Chau P L and Hardwick A J 1998 A new order parameter for tetrahedral configurations Mol. Phys. 93511

59. Luzar A and Chandler D 1996 Effect of environment on hydrogen bond dynamics in liquid water Phys. Rev. Lett. 76928 